\title{
An Overshoot-Constrained Fast Setpoint Control for Nanopositioning Systems with Switched Controllers
}

\author{
Zhizheng Wu $\mathbb{D}^{1},{ }^{1}$ Tengfei Yue, ${ }^{1}$ Xinxiang Jiang, ${ }^{1}$ Ning Cao $\left(\mathbb{D},{ }^{2}\right.$ Feng Li $\left(\mathbb{D},{ }^{3}\right.$ Fanfan Qian, \\ Merveilles Dziki, ${ }^{1}$ Zhu Zhang, ${ }^{4}$ Mei Liu, ${ }^{1}$ and Shaorong Xie ${ }^{1}$ \\ ${ }^{1}$ Department of Precision Mechanical Engineering, Shanghai University, Shanghai 200444, China \\ ${ }^{2}$ School of Mechanical and Electrical Engineering, Zhengzhou University of Light Industry, Zhengzhou 450002, China \\ ${ }^{3}$ School of Optical-Electrical and Computer Engineering, University of Shanghai for Science and Technology, \\ Shanghai 200093, China \\ ${ }^{4}$ Nanophotonics, Debye Institute for Nanomaterials Science, Utrecht University, 3508 TA Utrecht, Netherlands \\ Correspondence should be addressed to Ning Cao; ncao@zzuli.edu.cn
}

Received 23 March 2019; Accepted 17 July 2019; Published 27 August 2019

Academic Editor: Xuping Zhang

Copyright ( 2019 Zhizheng Wu et al. This is an open access article distributed under the Creative Commons Attribution License, which permits unrestricted use, distribution, and reproduction in any medium, provided the original work is properly cited.

\begin{abstract}
Nanopositioning control as the key technology has been applied in many fields such as near-field optics, biomedical engineering, and nanomanipulation, where it is required to possess high positioning accuracy, reliability, and speed. In this paper, a switched PID controller-based fast setpoint control method is proposed for nanopositioning systems. In order to improve the setpoint speed of the nanopositioning system without a large overshoot, a switched controller consisting of the approach mode and smooth mode is synthesized. The overshoot constraint of the resulting switched closed-loop system is investigated within a set of bilinear matrix inequalities, based on which the search of the controller parameters can be further processed by solving the properly formulated synthesis algorithm. The proposed control method is evaluated in a nanopositioning experimental system driven by a PZT actuator, and the experimental results demonstrate the effectiveness of the switched PID controller for the fast setpoint approaching operation.
\end{abstract}

\section{Introduction}

Nanomanipulation has been drawing continuous attention recently [1-3], among which nanopositioning technology as one of the key technologies is increasingly used for a wide range of applications, such as near-field optics system [4], scanning probe microscope [5], and micro-nano operation $[6,7]$. For example, in the near-field optics system, nanopositioning technology is used to operate the optic head toward the sample surface quickly and thus control the interaction between the lens and the samples precisely $[8,9]$. However, for achieving near-field operation successfully, not only the accuracy and positioning speed of the nanopositioning system are considered but also the overshoot in the positioning process should be balanced $[10,11]$. Generally, the response speed of the nanopositioning system should be fast enough to improve the efficiency. However, when the response speed of the closed-loop system is so fast, the output of the system is prone to have large overshoot, which leads to the collision between the nano-operated lens/ tip and the sample, thus resulting in their damage.

At present, various nanopositioning methods have been considered to meet the requirement of high precision and reliability in the control system [12, 13]. However, for conventional lead-lag controllers, such as proportional-integral controllers [14], although they are simple in structure and convenient in design, they are prone to have large overshoots and are difficult to meet the high-speed requirement [15]. In order to achieve high positioning speed, the $H_{\infty}$ controller can be designed to speed up the response of the system and improve the robustness of the system by establishing an auxiliary objective function based on the system's response speed [16]. Chen and Francis convert the rapid solution of positioning control into a closed-loop 
system $\mathrm{H}_{2}$ optimization problem by introducing a virtual integrator [17] and then using the classical $\mathrm{H}_{2}$ optimal controller to meet the requirements of high speed in the positioning control system. However, these high-speed positioning methods may bring out large overshoot for the closed-loop system and cause a collision with the samples in the nanopositioning systems.

To achieve the high-speed positioning operation with small overshoot in the nanopositioning system, many highspeed positioning control methods have been proposed to restrict the overshoot. An intelligent tuning method of proportional-integral-derivative (PID) parameters based on iterative learning control is proposed to self-adjust PID parameters of the atomic force microscope (AFM) according to the sample topography, thereby reducing overshoot and improving positioning accuracy of the system [18]. Kuiper and Schitter proposed a method for the tip-sample position using a model-based feedback control method to reduce the residual tracking error [19]; thus, the collision and the chance of damage of the tip and the sample are avoided. Kim et al. proposed a gap approaching method by using the acceleration feed-forward controller (AFC) to control and maintain the nanometer-scale disk/lens distance in a nearfield optics storage system [11], but the feed-forward reference signal measurement is not always accurate, which could reduce the high-speed performance of the positioning operation with small overshoot.

The aforementioned high-speed positioning control methods mostly adopted the control scheme of a single controller to improve the control speed under a satisfied overshoot in the nanopositioning systems. However, for the single controller, it must make a compromise between high positioning speed and low overshoot. In order to further improve the positioning speed and at the same time to restrict the overshoot, the control methods based on multiple controllers using switching rules have been proposed in the literature. Kim et al. proposed the mode-switching servo with a brake pulse [20] to reduce the initial overshoot and settling time. The mode-switching rule which consists of approach, hand-over, and gap-control modes with an optimal exponential reference input was adopted to further reduce the overshoot. Zimmermann et al. proposed a new positioning method based on timeto-digital converter (TDC) and used in scanning electron microscope [21], where the controller switching rules depend on positional information from the position sensor's feedback in closed-loop systems. A dual-stage positioning system is proposed by Zhu et al. to further reduce overshoot and improve positioning accuracy [22]. However, the selection of these switching rules to balance the speed and overshoot in the closed-loop system is mainly dependent on the designers' tryand-error experience, lacking corresponding theoretical analysis and controller synthesis methods.

In order to improve the positioning speed with a constrained overshoot in nanopositioning systems, in this paper, a switched PID controller-based fast setpoint approaching method is proposed to ensure high positioning speed within a limited overshoot. The controller design constraints are represented using a set of properly formulated bilinear matrix inequalities (BMIs). Then, a switched controller synthesis algorithm is proposed based on $\mathrm{H}_{2}$-optimized performance under the overshoot constraints. The performance of the switched controllers is experimentally evaluated in a near-field optics positioning system driven by a PZT actuator, and the experimental results are presented to illustrate the effectiveness of the proposed fast setpoint control method.

\section{Switched PID Controller with Overshoot Constraint}

2.1. Closed-Loop System with Switched PID Controller. Consider the model of the plant given in state-space form as

$$
\Sigma_{p}:\left\{\begin{array}{l}
x_{p}(k+1)=A_{p} x_{p}(k)+B_{p} u(k), \\
y(k)=C_{p} x_{p}(k), \\
e(k)=-C_{p} x_{p}(k)+r
\end{array}\right.
$$

where $A_{p} \in R^{n \times n}, B_{p} \in R^{n \times 1}$, and $C_{p} \in R^{1 \times n}$ are the coefficient matrices; $x_{p}(k) \in R^{n}$ is the state vector; $u(k)$ is the control input; $y(k)$ is a measurement signal to be fed back to the controller; $e(k)$ is the performance variable to be regulated; and $r$ is the positioning reference input signal.

The switched PID controller, which consists of the approach and smooth control mode, can be expressed as

$$
\begin{aligned}
& C_{c}^{1}(z)=K_{c}^{p 1}+K_{c}^{i 1} \frac{z}{z-1}+K_{c}^{d 1} \frac{z-1}{z}, \\
& C_{c}^{2}(z)=K_{c}^{p 2}+K_{c}^{i 2} \frac{z}{z-1}+K_{c}^{d 2} \frac{z-1}{z} .
\end{aligned}
$$

The corresponding state-space realization of $\Sigma_{c j}$ can be written as

$$
\Sigma_{c j}:\left\{\begin{array}{l}
x_{c}(k+1)=A_{c j} x_{c}(k)+B_{c j} e(k), \\
u(k)=C_{c j} x_{c}(k)+D_{c j} e(k), \\
j= \begin{cases}1, & \text { if } e \geq N_{0}, \\
2, & \text { if } e<N_{0},\end{cases}
\end{array}\right.
$$

where $A_{c j}=\left[\begin{array}{ll}1 & 1 \\ 0 & 0\end{array}\right], \quad B_{c j}=\left[\begin{array}{c}K_{c}^{i j}-K_{c}^{d j} \\ K_{c}^{d j}\end{array}\right], \quad C_{c j}=\left[\begin{array}{ll}1 & 0\end{array}\right]$, $D_{c j}=\left[K_{c}^{p j}+K_{c}^{i j}+K_{c}^{d j}\right]$, and $j \in\{1,2\}$ and $N_{0}$ is the value of the switching surface. Let $x(k)=\left[x_{p}(k) x_{c}(k)\right]^{T} \in R^{n+2}$, then combining (1) and (4) yields the following closed-loop system:

$$
\sum_{s j}\left\{\begin{array}{l}
x(k+1)=A_{s j} x(k)+B_{s j} r, \\
e(k)=C_{s j} x(k)+r, \\
j= \begin{cases}1, & \text { if } e(k)=C_{s j} x(k)+r \geq N_{0}, \\
2, & \text { if } e(k)=C_{s j} x(k)+r<N_{0},\end{cases}
\end{array}\right.
$$

where $A_{s j}=\left[\begin{array}{cc}A_{p}-B_{p} D_{c j} C_{p} & B_{p} C_{c j} \\ -B_{c j} C_{p} & A_{c j}\end{array}\right], B_{s j}=\left[\begin{array}{c}B_{p} D_{c j} \\ B_{c j}\end{array}\right]$, and $C_{s j}=\left[\begin{array}{ll}-C_{p} & 0\end{array}\right]$.

The main idea of the controller design is to define a switching surface that when the system parameters satisfy 
$e \leq N_{0}, \Sigma_{s 1}$ will be converted into $\Sigma_{s 2}$ such that the control speed and overshoot constraint are satisfied. The controller parameters can be determined in the scope of a set of properly formulated BMIs, as outlined in the following sections.

2.2. Constraint for the Overshoot. In this section, the problem of the switched PID controller design with a restrict constraint for the overshoot on the closed-loop system is investigated. Based on the closed-loop system represented with the switched PID controllers as in (5), the following conditions can be used to design the controller parameters with respect to the overshoot constraint.

Theorem 1. Consider the switched closed-loop system (5) where the matrix $B_{p}$ is full-rank matrix with initial state $x(0)=0$ and $0<r_{1}<r<r_{2}, \delta>0$. Let $\kappa=\max \left|\|r\|^{2}\right|=r_{2}^{2}$, $\widetilde{N}_{0}=N_{0}-r_{2}, \tilde{\delta}=-\delta-r_{1}, \quad j \in\{1,2\}$, and $m \in\{1,2,3,4\}$. $\alpha>0$ and $\beta>0$ are preset constants. If the existence of symmetric matrices $P>0, A_{0}>0$, vector $q, b_{0}$, scalar $g, c_{0}, \mu, \tau_{m} \geq 0$, and $\alpha g \leq \mu$ satisfies the following inequality (6)-(10), then the performance e of the closed-loop system will be bounded with $e>-\delta$ and the switching from $\Sigma_{s 1}$ to $\Sigma_{s 2}$ only happens once.

$\left[\begin{array}{ll}P & q \\ q^{T} & g\end{array}\right]>0$

$\left[\begin{array}{cccc}-P+\alpha P & A_{s j}^{T} q+(\alpha-1) q & 0 & A_{s j}^{T} P \\ q^{T} A_{s j}+(\alpha-1) q^{T} & \alpha g & q^{T} B_{s j} & 0 \\ 0 & B_{s j}^{T} q & -\frac{\mu}{\kappa} I & B_{s j}^{T} P \\ P A_{s j} & 0 & P B_{s j} & -P\end{array}\right] \leq 0$,

$\left[\begin{array}{cc}A_{0}-\beta P & b_{0}-\beta q-\tau_{1} C_{s 2}^{T}+\tau_{2} C_{s 2}^{T} \\ b_{0}^{T}-\beta q^{T}-\tau_{1} C_{s 2}+\tau_{2} C_{s 2} & c_{0}-\beta\left(g-\frac{\mu}{\alpha}\right)+2 \tau_{1} \tilde{N}_{0}-2 \tau_{2} \widetilde{\delta}\end{array}\right] \leq 0$,

$\left[\begin{array}{cc}-A_{0} & -b_{0}+\tau_{3} A_{s 2}^{T} C_{s 2}^{T} \\ -b_{0}^{T}+\tau_{3} C_{s 2} A_{s 2} & -c_{0}+2 \tau_{3} C_{s 2} B_{s 2} r_{2}-2 \tau_{3} \tilde{N}_{0}\end{array}\right] \leq 0$,

$\left[\begin{array}{cc}-A_{0} & -b_{0}-\tau_{4} A_{s 2}^{T} C_{s 2}^{T} \\ -b_{0}^{T}-\tau_{4} C_{s 2} A_{s 2} & -c_{0}-2 \tau_{4} C_{s 2} B_{s 2} r_{1}+2 \tau_{4} \tilde{\delta}\end{array}\right] \leq 0$

Proof. The state-space representation of the closed-loop system is given by formula (5), and a quadratic function $V(x)=x^{T} P x+2 q^{T} x+g$ is constructed. First, multiplying by $\left[\begin{array}{c}x \\ 1\end{array}\right]^{T}$ and $\left[\begin{array}{l}x \\ 1\end{array}\right]$ from the left-hand and right-hand sides of (6), we have $V(x)=x^{T} P x+2 q^{T} x+g>0$. Based on (7), the following inequality (11) is obtained by using the Schur complement formula:

$$
\begin{gathered}
{\left[\begin{array}{ccc}
A_{s j}^{T} P A_{s j}-P+\alpha P & A_{s j}^{T} q+(\alpha-1) q & A_{s j}^{T} P B_{s j} \\
q^{T} A_{s j}+(\alpha-1) q^{T} & \alpha g & q^{T} B_{s j} \\
B_{s j}^{T} P A_{s j} & B_{s j}^{T} q & B_{s j}^{T} P B_{s j}-\frac{\mu}{\kappa} I
\end{array}\right] \leq 0 .} \\
\text { Multiplying by }\left[\begin{array}{c}
x(k) \\
1 \\
r
\end{array}\right]^{T} \text { and }\left[\begin{array}{c}
x(k) \\
1 \\
r
\end{array}\right] \text { from the left-hand }
\end{gathered}
$$

and right-hand sides of (11), respectively, we have

$$
V(x(k+1)) \leq \frac{\mu}{\kappa} r^{2}+(1-\alpha) V(x(k)) .
$$

It follows from (12) that

$$
V(x(k+1)) \leq \mu+(1-\alpha) V(x(k)) .
$$

When $\alpha V(x(k)) \geq \mu$ is satisfied, then we have

$$
V(x(k+1))-V(x(k))<0 .
$$

Based on the inequality (13) and (14), $V(x(k))$ will finally decrease to

$$
V(x(k))=x^{T} P x+2 q^{T} x+g \leq \frac{\mu}{\alpha}, \quad \forall k \geq 0 .
$$

Based on the inequality (15), we define an ellipsoid that

$$
\varepsilon=\left\{x \mid x^{T} P x+2 q^{T} x+g-\frac{\mu}{\alpha} \leq 0\right\} .
$$

Assume that the initial state is $x(0)=0$, since the scalars satisfy $\alpha g \leq \mu$, we then have $V(0)=g \leq \mu / \alpha$. Therefore, inequality (15) implies that the state $x$ of the switched closedloop system always involves inside the ellipsoid $\varepsilon$. If the subspace inside the ellipsoid $\varepsilon$, which satisfies $-\delta<e(k)<N_{0}$, is an invariant set $\widetilde{\varepsilon}$, then we can conclude that the switching from $\Sigma_{s 1}$ to $\Sigma_{s 2}$ only happens once and $e>-\delta$ for $\forall k \geq 0$.

Based on the closed-loop system (5), it is obvious that for the state $x$ in $\widetilde{\varepsilon}$ if

$$
\begin{gathered}
-\delta-r<C_{s 2} x(k)<N_{0}-r \\
-\delta-\left(C_{s 2} B_{s 2}+1\right) r<C_{s 2} A_{s 2} x(k)<N_{0}-\left(C_{s 2} B_{s 2}+1\right) r
\end{gathered}
$$

are satisfied, then $-\delta<e(k)<N_{0}$ always holds after the switching surface is crossed. Since $0<r_{1}<r<r_{2}$, define $\tilde{N}_{0}=$ $N_{0}-r_{2}$ and $\widetilde{\delta}=-\delta-r_{1}$; then, conditions (17) and (18) can be simplified to

$$
\begin{aligned}
& \widetilde{\delta}<C_{s 2} x(k)<\tilde{N}_{0}, \\
& \widetilde{\delta}-C_{s 2} B_{s 2} r_{1}<C_{s 2} A_{s 2} x(k)<\tilde{N}_{0}-C_{s 2} B_{s 2} r_{2} .
\end{aligned}
$$

We define the following subspace: 


$$
\begin{aligned}
& \varepsilon_{0}=\left\{x \mid x^{T} A_{0} x+2 x^{T} b_{0}+c_{0} \leq 0\right\}, \\
& \varepsilon_{1}=\left\{x \mid C_{s 2} x-\widetilde{N}_{0} \leq 0\right\}, \\
& \varepsilon_{2}=\left\{x \mid-C_{s 2} x+\widetilde{\delta} \leq 0\right\}, \\
& \varepsilon_{3}=\left\{x \mid C_{s 2} A_{s 2} x+C_{s 2} B_{s 2} r_{2}-\tilde{N}_{0} \leq 0\right\}, \\
& \varepsilon_{4}=\left\{x \mid-C_{s 2} A_{s 2} x-C_{s 2} B_{s 2} r_{1}+\widetilde{\delta} \leq 0\right\} .
\end{aligned}
$$

If the ellipsoid $\varepsilon_{0}$ covers the intersection of ellipsoids $\varepsilon, \varepsilon_{1}$, and $\varepsilon_{2}$ but is contained inside the intersection of $\varepsilon_{3}$ and $\varepsilon_{4}$, namely,

$$
\begin{aligned}
& \mathcal{E}_{0} \supseteq \varepsilon \cap \varepsilon_{1} \cap \varepsilon_{2}, \\
& \varepsilon_{3} \supseteq \varepsilon_{0}, \\
& \varepsilon_{4} \supseteq \varepsilon_{0},
\end{aligned}
$$

are satisfied, then the subspace $\widetilde{\varepsilon}$ is invariant. By the $\mathrm{S}$ procedure, (21) is true if there exist nonnegative scalars $\beta$, $\tau_{1}, \tau_{2}, \tau_{3}$, and $\tau_{4}$ such that

$$
\begin{aligned}
& {\left[\begin{array}{ll}
A_{0} & b_{0} \\
b_{0}^{T} & c_{0}
\end{array}\right]-\beta\left[\begin{array}{cc}
P & q \\
q^{T} & g-\frac{\mu}{\alpha}
\end{array}\right]-\tau_{1}\left[\begin{array}{cc}
0 & C_{s 2}^{T} \\
C_{s 2} & -2 \tilde{N}_{0}
\end{array}\right]} \\
& \quad-\tau_{2}\left[\begin{array}{cc}
0 & -C_{s 2}^{T} \\
-C_{s 2} & 2 \widetilde{\delta}
\end{array}\right] \leq 0, \\
& \quad-\left[\begin{array}{ll}
A_{0} & b_{0} \\
b_{0}^{T} & c_{0}
\end{array}\right]+\tau_{3}\left[\begin{array}{cc}
0 & A_{s 2}^{T} C_{s 2}^{T} \\
C_{s 2} A_{s 2} & 2 C_{s 2} B_{s 2} r_{2}-2 \tilde{N}_{0}
\end{array}\right] \leq 0, \\
& -\left[\begin{array}{ll}
A_{0} & b_{0} \\
b_{0}^{T} & c_{0}
\end{array}\right]+\tau_{4}\left[\begin{array}{cc}
0 & -A_{s 2}^{T} C_{s 2}^{T} \\
-C_{s 2} A_{s 2} & -2 C_{s 2} B_{s 2} r_{1}+2 \widetilde{\delta}
\end{array}\right] \leq 0 .
\end{aligned}
$$

Based on (22)-(24), the inequalities (8)-(10) can be derived. Therefore, if (6)-(10) in Theorem 1 can be satisfied, then the subspace $\widetilde{\mathcal{E}}$ is invariant, which means that the switching from $\Sigma_{s 1}$ to $\Sigma_{s 2}$ only happens once and $e>-\delta$ for $\forall k \geq 0$.

It shows that the proposed switched PID controller can control the nanopositioning system to restrict the overshoot and realize the fast setpoint approaching to the desired position when Theorem 1 is satisfied.

2.3. $\mathrm{H}_{2}$ Performance Formulation. In this section, the problem of the switched PID controller design with $\mathrm{H}_{2}$ performance for $\Sigma_{s 2}$ on the closed-loop system is investigated. Since the switching only happens once, for the optimization of $\mathrm{H}_{2}$ performance, we only consider $\Sigma_{s 2}$ after switching. In the following, the convergence rate of the closed-loop system with respect to the static inputs is performed by minimizing $\mathrm{H}_{2}$ performance specification.
Consider the nominal closed-loop system subject to an input $r$. An additional controller design objective is considered where it is desired to find a PID controller that minimizes the $l_{2}$ norm of $e$, that is, $\|e\|_{2}$, by considering only the nominal system in the closed-loop system. Since the sensitivity function $S_{0}$ relates the input $r$ to the error $e$, the solution of this design constraint can be obtained by considering a standard $\mathrm{H}_{2}$ optimal control problem where it is desired to minimize the $H_{2}$ norm of the system $z /(z-1) S_{0}$. In order to avoid the unstable pole introduced by $z /(z-1)$, the system $z /(z-1) S_{0}$ can be approximated by the system $z /\left(z-1+\alpha_{0}\right) S_{0}$, where $\alpha_{0}$ is a small positive constraint. Therefore, the state-space equation of the integral part introduced is

$$
\sum_{z}:\left\{\begin{array}{l}
x_{z}(k+1)=A_{z} x_{z}(k)+B_{z} \varphi(k) \\
y_{z}(k)=C_{z} x_{z}(k)+D_{z} \varphi(k)
\end{array}\right.
$$

where $A_{z}=C_{z}=1-\alpha_{0}, B_{z}=D_{z}=1$, and $x_{z}(k) \in R$ are state vectors and $\varphi(k)$ is the pulse input signal.

Let $x(k)=\left[x_{z}(k) x_{p}(k) x_{c}(k)\right]^{T} \in R^{n+3}$, then the closed-loop system (5) can be converted to

$$
\sum_{H}\left\{\begin{array}{l}
x(k+1)=A_{H} x(k)+B_{H} \varphi(k), \\
e(k)=C_{H} x(k)+D_{H} \varphi(k),
\end{array}\right.
$$

where $\quad A_{H}=\left[\begin{array}{ccc}A_{z} & 0 & 0 \\ B_{p} D_{c 2} C_{z} & A_{p}-B_{p} D_{c 2} C_{p} & B_{p} C_{c 2} \\ B_{c 2} C_{z} & -B_{c 2} C_{p} & A_{c 2}\end{array}\right], \quad B_{H}=$ $\left[\begin{array}{c}B_{z} \\ B_{p} D_{c 2} D_{z} \\ B_{c 2} D_{z}\end{array}\right], C_{H}=\left[\begin{array}{lll}C_{z}-C_{p} & 0\end{array}\right]$, and $D_{H}=D_{z}$.

Let $\widetilde{A}_{H}=\left[\begin{array}{ccc}A_{z} & 0 & 0 \\ 0 & A_{p} & B_{p} C_{c 2} \\ 0 & 0 & A_{c 2}\end{array}\right], \quad \widetilde{B}_{H}=\left[\begin{array}{c}B_{z} \\ 0 \\ 0\end{array}\right], \quad$ and $K=$ $\left[\begin{array}{c}D_{c 2} \\ B_{c 2}\end{array}\right]$. Since $B_{p}$ is assumed to be of full-column rank, there exists a nonsingular transformation matrix $T_{b} \in R^{n \times n}$ such that $T_{b} B_{p}=\left[\begin{array}{l}0 \\ 1\end{array}\right]_{n \times 1}$. Let $T=\left[\begin{array}{ccc}1 & 0 & 0 \\ 0 & T_{b} & 0 \\ 0 & 0 & I_{2 \times 2}\end{array}\right] \in R^{N \times N}$, $N=n+3$. Based on the closed-loop system represented with the switched PID controllers as in (26), the following LMIs conditions can be used to design the controller parameters in $\mathrm{C}_{c}^{2}$ with respect to $H_{2}$ performance.

Theorem 2. Consider the closed-loop system (26) where the matrix $B_{p}$ is full-rank matrix. If there exist a positive definite matrix $Q \in R^{N \times N}, J=\left[\begin{array}{cc}J_{11} & 0 \\ J_{21} & J_{22}\end{array}\right] \in R^{N \times N}, J_{22} \in R^{3 \times 3}, L_{1}=$ $\left[\begin{array}{ll}L_{11} & 0\end{array}\right] \in R^{N \times N}, \quad L_{11} \in R^{N \times(N-n-2)}, \quad L_{2}=\left[\begin{array}{ll}L_{21} & 0\end{array}\right] \in R^{1 \times N}$, $L_{21} \in R^{1 \times(N-n-2)}, Y=\left[C_{z}-C_{p}\right], Z \in R^{3 \times 1}$, and $\gamma>0$ such that (27)-(29) are satisfied: 


$$
\left[\begin{array}{ccc}
Q-J T-T^{T} J^{T} & N_{1}-T^{T} L_{1}^{T}+J T \widetilde{A}_{H} & N_{2}-T^{T} L_{2}^{T}+J T \widetilde{B}_{H} \\
N_{1}^{T}-L_{1} T+\widetilde{A}_{H}^{T} T^{T} J^{T} & -Q+L_{1} T \widetilde{A}_{H}+\widetilde{A}_{H}^{T} T^{T} L_{1}^{T} & \widetilde{A}_{H}^{T} T^{T} L_{2}^{T}+L_{1} T \widetilde{B}_{H} \\
N_{2}^{T}-L_{2} T+\widetilde{B}_{H}^{T} T^{T} J^{T} & L_{2} T \widetilde{A}_{H}+\widetilde{B}_{H}^{T} T^{T} L_{1}^{T} & -I+L_{2} T \widetilde{B}_{H}+\widetilde{B}_{H}^{T} T^{T} L_{2}^{T}
\end{array}\right]<0
$$

$$
\left[\begin{array}{ccc}
Q & 0 & C_{H}^{T} \\
0 & I & D_{H}^{T} \\
C_{H} & D_{H} & S
\end{array}\right]>0
$$

$$
\operatorname{Tr}(S)<\gamma
$$

where $N_{1}=\left[\begin{array}{cc}0 & 0 \\ Z Y & 0\end{array}\right]$ and $N_{2}=\left[\begin{array}{c}0 \\ Z D_{z}\end{array}\right]$, and if there exists $K$ such that

$$
J_{22} K=Z
$$

then the $\mathrm{H}_{2}$ norm of the closed-loop system (26) satisfies $\left\|\Sigma_{H}\right\|_{2}<\gamma$.

Proof. First, by simple calculation, inequality (27) is equivalent to

$$
\begin{aligned}
& {\left[\begin{array}{ccc}
Q & N_{1} & N_{2} \\
N_{1}^{T} & -Q & 0 \\
N_{2}^{T} & 0 & -I
\end{array}\right]+\left[\begin{array}{c}
J \\
L_{1} \\
L_{2}
\end{array}\right] T\left[\begin{array}{lll}
-I_{N \times N} & \widetilde{A}_{H} & \widetilde{B}_{H}
\end{array}\right]} \\
& +\left[\begin{array}{ccc}
-I_{N \times N} & \widetilde{A}_{H} & \widetilde{B}_{H}
\end{array}\right]^{T} T^{T} \\
& \quad \cdot\left[\begin{array}{c}
J \\
L_{1} \\
L_{2}
\end{array}\right]^{T}<0 .
\end{aligned}
$$

Using $J_{22} K=Z$ from (30), we have $N_{1}=$ $\left[\begin{array}{cc}0_{n \times(n+1)} & 0_{n \times 2} \\ Z Y & 0_{(N-n) \times 2}\end{array}\right]=J T\left[\begin{array}{ccc}0 & 0 & 0 \\ B_{p} D_{c 2} C_{z} & -B_{p} D_{c 2} C_{p} & 0 \\ B_{c 2} C_{z} & -B_{c 2} C_{p} & 0\end{array}\right]$ and $N_{2}=\left[\begin{array}{c}0 \\ Z D_{z}\end{array}\right]=J T\left[\begin{array}{c}0 \\ B_{p} D_{c 2} \\ B_{c 2}\end{array}\right]$. Then, we also have that $N_{1}+J T \widetilde{A}_{H}=J T A_{H}, N_{2}+J T \widetilde{B}_{H}=J T B_{H}, L_{i} T \widetilde{A}_{H}=L_{i} T A_{H}$, and $L_{i} T \widetilde{B}_{H}=L_{i} T B_{H}, i=1,2$. It follows from (31) that

$$
\begin{aligned}
& {\left[\begin{array}{ccc}
Q & 0 & 0 \\
0 & -Q & 0 \\
0 & 0 & -I
\end{array}\right]+\left[\begin{array}{c}
J \\
L_{1} \\
L_{2}
\end{array}\right] T\left[\begin{array}{lll}
-I_{N \times N} & A_{H} & B_{H}
\end{array}\right]} \\
& +\left[\begin{array}{lll}
-I_{N \times N} & A_{H} & B_{H}
\end{array}\right]^{T} T^{T} \\
& \quad\left[\begin{array}{c}
J \\
L_{1} \\
L_{2}
\end{array}\right]^{T}<0 .
\end{aligned}
$$

$$
\text { Multiplying by }\left[\begin{array}{c}
x(k+1) \\
x(k) \\
\varphi(k)
\end{array}\right]^{T} \text { and }\left[\begin{array}{c}
x(k+1) \\
x(k) \\
\varphi(k)
\end{array}\right] \text { from the }
$$

left-hand and right-hand sides of (32), we have

$$
x(k+1)^{T} \mathrm{Q} x(k+1)<x(k)^{T} \mathrm{Q} x(k)+\varphi(k)^{T} \varphi(k),
$$

which is equivalent to

$$
\left[\begin{array}{ccc}
-Q & 0 & A_{H}^{T} Q \\
0 & -I & B_{H}^{T} Q \\
Q A_{H} & Q B_{H} & -Q
\end{array}\right]<0
$$

Consider a congruence transformation on (34) and (28), where $X=Q^{-1}$, then we have

$$
\begin{aligned}
& {\left[\begin{array}{ccc}
-X & X A_{H} & 0 \\
A_{H}^{T} X & -X & B_{H}^{T} \\
0 & B_{H} & -I
\end{array}\right]<0,} \\
& {\left[\begin{array}{ccc}
X & 0 & X C_{H} \\
0 & I & D_{H} \\
C_{H}^{T} X & D_{H}^{T} & S
\end{array}\right]>0,}
\end{aligned}
$$

Hence, the design of $\Sigma_{H}$ that satisfies a closed-loop $H_{2}$ norm constraint [17] can be performed with the LMIs (27)-(29).

2.4. Switched Controller Synthesis. In this section, the switched controller synthesis on the closed-loop system is investigated, and the corresponding synthesis algorithm based on the matrix inequalities in Sections 2.2 and 2.3 is summarized as follows.

Step 1. Initialize the $\Sigma_{s 1}$ controller parameters $K_{c}^{p 1}, K_{c}^{i 1}$, and $K_{c}^{d 1}$ to obtain a high-speed response for the nanopositioning system without considering the influence of overshoot. Define a switching surface $e=N_{0}, N_{0} \in\left[N_{m}, N_{n}\right]$, where $N_{m}$ and $N_{n}$ are the given switching surface threshold, so that the system transits to the smooth positioning mode controller after crossing the switching surface. Let $D_{c 2}^{\ell, j}, B_{c 2}^{\ell, j}, g^{\ell, j}$, $\mu^{\ell, j}, A_{0 .}^{\ell, j}, b_{0}^{\ell, j}, c_{0}^{\ell, j}, Q^{\ell, j}, J_{22}^{\ell, j}, Z^{\ell, j}, L_{11}^{\ell, j}, L_{21}^{\ell, j}, S^{\ell, j}, \gamma^{\ell, j}, P^{\ell, j}, q^{\ell, j}$, and $\tau_{m}^{\ell, j}$ denote the values of $D_{c 2}, B_{c 2}, g, \mu, A_{0}, b_{0}, c_{0}, Q, J, Z$, $L_{11}, L_{21}, S, \gamma, P, q$, and $\tau_{m}$ in the $e^{\text {th }}$ step and $j^{\text {th }}$ step in the proposed iterative synthesis algorithm.

Step 2. Set the initial switching surface value be $N_{m}$, let $N_{0}^{j}=N_{m}, j=0$. 
Step 3. Start the $j^{\text {th }}$ step: at the $j^{\text {th }}$ iteration, $j=0,1,2, \ldots$, calculate the initial values of $D_{c 2}^{0, j}$ and $B_{c 2}^{0, j}$ by formulae (6)-(10). If there is a feasible solution, then go to Step 4. Otherwise, let $N_{0}^{j+1}=N_{0}^{j}+\Delta N_{0}$, where $\Delta N_{0}$ is chosen to be small. If $N_{0}^{j+1} \leq N_{n}$, return to Step 3. Else, go to Step 6 .

Step 4. Start the $\ell^{\text {th }}$ step:

(i) At the $\ell^{\text {th }}$ iteration, $\ell=1,2, \ldots$, minimize $\gamma^{\ell, j}$ subject to (6)-(10) and (27)-(30) with unknown $P^{\ell, j}, q^{\ell, j}$, $\tau_{m}^{\ell, j}, g^{\ell, j}, \mu^{\ell, j}, A_{0}^{\ell, j}, b_{0}^{\ell, j}, c_{0}^{\ell, j}, Q^{\ell, j}, J_{22}^{\ell, j}, Z^{\ell, j}, L_{11}^{\ell, j}, L_{21}^{\ell, j}$, $S^{\ell, j}$, and $\gamma^{\ell, j}$ and with known $D_{c 2}^{\ell-1, j}$ and $B_{c 2}^{\ell-1, j}$.

(ii) At the $\ell^{\text {th }}$ iteration, $\ell=1,2, \ldots$, minimize $\gamma^{\ell, j}$ subject to (6)-(10) and (27)-(30) with unknown $D_{c 2}^{\ell, j}, B_{c 2}^{\ell, j}$, $g^{\ell, j}, \mu^{\ell, j}, A_{0}^{\ell, j}, b_{0}^{\ell, j}, c_{0}^{\ell, j}, Q^{\ell, j}, J_{22}^{\ell, j}, Z^{\ell, j}, L_{11}^{\ell, j}, L_{21}^{\ell, j}, S^{\ell, j}$, and $\gamma^{\ell, j}$ and with known $P^{\ell, j}, q^{\ell, j}$, and $\tau_{m}^{\ell, j}$.

(iii) If $\left|\gamma^{\ell-1, j}-\gamma^{\ell, j}\right|<\sigma_{\beta}$, where $\sigma_{\beta}$ is a prescribed tolerance, record $\gamma^{\ell, j}, D_{c 2}^{\ell, j}$, and $B_{c 2}^{\ell, j}$, stop iterative loop, and go to Step 5. Else return to Step 4 .

Step 5. Let $\gamma_{c}^{j}=\gamma^{\ell, j}, \quad D_{c 2}^{j}=D_{c 2}^{\ell, j}$, and $B_{c 2}^{j}=B_{c 2}^{\ell, j}$ and let $N_{0}^{j+1}=N_{0}^{j}+\Delta N_{0}$, where $\Delta N_{0}$ is chosen to be small. If $N_{0}^{j+1} \leq N_{n}$, return to Step 3. Else, go to Step 6 .

Step 6. Select the minimum value in the array of $\gamma_{c}^{j}$ with the corresponding $D_{c 2}^{j}$ and $B_{c 2}^{j}$. Then, based on equations (3) and (4), the values of $K_{c}^{p 2}, K_{c}^{i 2}$, and $K_{c}^{d 2}$ in the controller $C_{c}^{2}$ can be obtained.

If the above synthesis algorithm yields solution, then the switched PID controller will be properly designed. It should be noted that the switched controller design approach is theoretically developed based on the closed-loop system model (5) and does not depend on specific hardware or a system. Therefore, for different applications, the system model (5) with the desired overshoot constraint should be properly formulated first.

Since the overshoot constraint formulated in Theorem 1 can be applied to the general closed-loop systems with different controller structures, the idea of the switched controller design approach can also be extended to other kind of controllers, for example, the dynamical output feedback controller. However, the controller synthesis algorithm needs to be reformulated according to different controller structures.

\section{Experimental Results}

In this section, the fast anticontact approaching method based on the switched PID controller is experimentally tested in a near-field optics positioning system driven by the PZT actuator. Experimental results are presented to show the performance of the resulting closed-loop system with the designed switched PID controller.

The schematic diagram of the experimental setup of the near-field optics positioning system is shown in Figure 1, where the optic lens needs to quickly move from the initial position to the point with a gap of $100 \mathrm{~nm}$ above the sample. The picture of the corresponding nanopositioning experimental system is represented in Figure 2. A multilayer PZT actuator (model PL 112.11, Physik Instrumente) is attached to the suspension beam and serves to adjust the position of the beam tip. The PZT actuator is driven by a power amplifier (E-650 LVPZT amplifier, Physik Instrumente). The real-time measurement of the tip position is performed using a laser Doppler interferometer (Polytec OFV-072, OFV-552, and OFV-5000). A PCI 6221 input-output card from National Instruments and a personal computer are used to implement the controller and to interface it with the rest of the system. The control algorithm is implemented using the real-time module and simulation interface toolkit with Matlab Simulink.

In order to facilitate the design of the switched PID controller, the distance error $e$ fed to the controller is defined as

$$
e=r-y
$$

where $r$ represents the initial distance between the near-field optic lens and the desired position and $e$ indicates the actual distance between the near-field optic lens and the desired position.

Using the Matlab System Identification Toolbox, the control system with the PZT actuator is first identified as a fourth-order system:

$$
y(k)=\frac{B\left(z^{-1}\right)}{A\left(z^{-1}\right)} u(k),
$$

where $\quad A\left(z^{-1}\right)=1-3.9084 z^{-1}+5.732 z^{-2}-3.7386 z^{-3}+$ $0.9150 z^{-4}$ and $B\left(z^{-1}\right)=0.0038 z^{-1}-0.0118 z^{-2}+0.0125 z^{-3}$ $-0.0044 z^{-4}$

In the following, based on the obtained model of the nanopositioning system, the initial distance between the sample and the near-field optic lens is set as $700 \mathrm{um}$. The ratio of the voltage and displacement of the interferometer is set as $0.01 \mathrm{~V} / \mathrm{um}$; therefore, the given reference signal $r$ is $7 \mathrm{~V}$. The control of nanopositioning system needs to consider the overshoot to avoid the collision, while ensuring high positioning speed.

Since the system (37) is a nonminimum phase system, it is normally difficult to find a single PID controller that can achieve a fast speed with limited overshoot. In the following, the traditional PID controller is first evaluated with the MATLAB PID Tuner toolbox under the overshoot constraint. The obtained fastest response of the closed-loop system with the satisfied overshoot limit is shown in Figure 3, where the system takes more than 0.4 seconds to fully settle down. By introducing a virtual integral in the system, the $\mathrm{H}_{2}$ controller, as a kind of optimal controller, can be designed to maximize the system track performance [17]. Therefore, the design of the $\mathrm{H}_{2}$ optimal controller is also evaluated to control the motion of the optic lens. Based on the MATLAB robust control toolbox, the $\mathrm{H}_{2}$ optimal controller is obtained by using the h2syn function as 


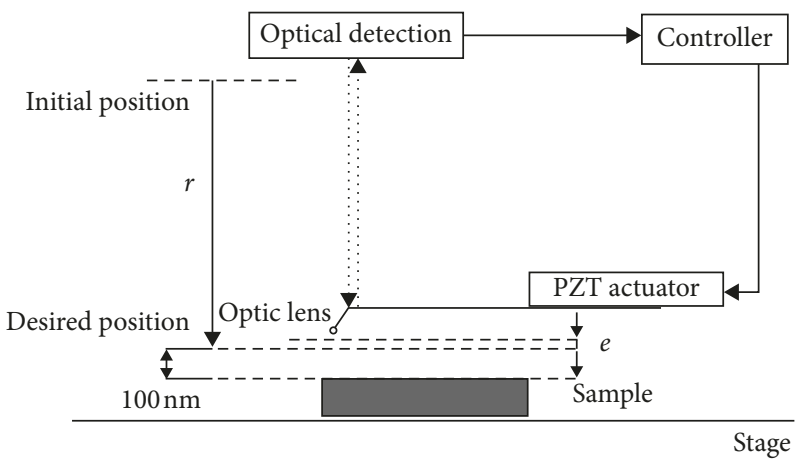

FIGURE 1: Schematic diagram of near-field optics positioning setup system.

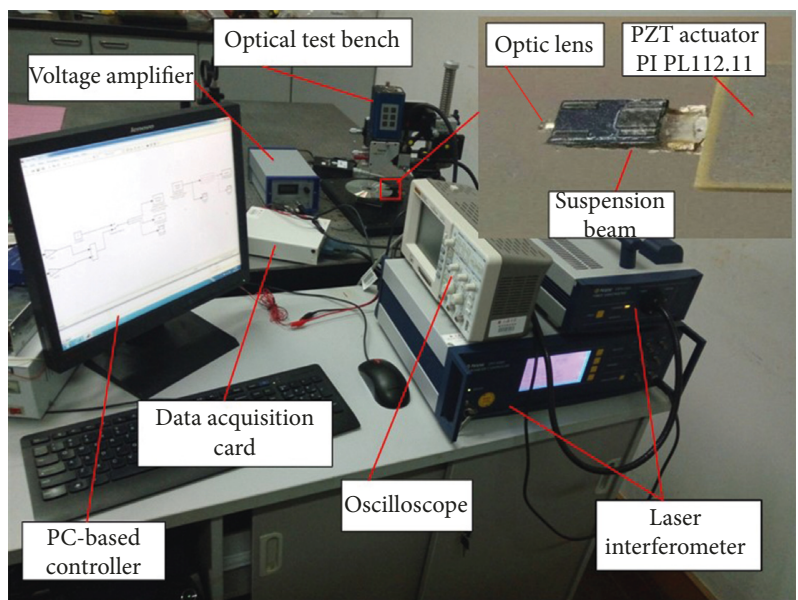

FIGURE 2: The experimental setup of near-field optics positioning system.

$$
K_{a}=\left[\begin{array}{ccccc}
0.728 & -0.2733 & 0.07004 & 0.06834 & -0.1401 \\
1.809 & 1.819 & 0.03398 & -0.4547 & 0.432 \\
1.187 e-11 & 4 & -3.057 e-12 & -2.983 e-12 & 6.115 e-12 \\
9.895 e-12 & 9.944 e-12 & 1 & -2.486 e-12 & 5.096 e-12 \\
4.947 e-12 & 4.972 e-12 & -1.1274 e-12 & 0.5 & 2.548 e-12
\end{array}\right],
$$$$
K_{b}=\left[\begin{array}{c}
62.5 \\
-1.013 e-9 \\
-2.728 e-9 \\
2.274 e-9 \\
-1.137 e-9
\end{array}\right]
$$

$$
K_{c}=\left[\begin{array}{c}
14.47 \\
-16.72 \\
11.74 \\
11.74 \\
7.116
\end{array}\right]^{T}
$$

$$
K_{d}=0 \text {. }
$$

Based on the obtained controller, the positioning response of the $\mathrm{H}_{2}$ optimized performance controller is evaluated in the nanopositioning system, as shown in Figure 4. 
It can be seen from Figure 4 that the $\mathrm{H}_{2}$ optimized controller tracks the step response quickly, and the adjust time is around $0.05 \mathrm{~s}$, and it takes $0.2 \mathrm{~s}$ to be fully stabilized. The rapid response capability of the closed-loop system is obtained, but the overshoot is large, which is not allowed for the nanopositioning system.

In order to deal with the overshoot shortcoming of a single $\mathrm{H}_{2}$ controller, in the following, the switched PID controllers, which include a fast approaching mode and a smooth transition mode with a switching surface to achieve a fast setpoint approaching, are designed and evaluated. First, the approach mode PID controller with a rise time of $0.1 \mathrm{~s}$ is designed to aim at a fast approach positioning to the sample and is represented as

$$
C_{c}^{1}(z)=0.006097+0.012194 \frac{z}{z-1}+0.005874 \frac{z-1}{z} .
$$

The simulation and experimental results of the closedloop system with the single approach mode PID controller are shown in Figure 5, which shows a large overshoot of more than $10 \%$.

Based on the proposed synthesis algorithm, the following steps are used to solve the parameters of the smooth mode controller. With the controller parameters $K_{c}^{p 1}=0.006097$, $K_{c}^{i 1}=0.012194$, and $K_{c}^{d 1}=0.005874$ and the preset constants $\delta=0.1, r_{1}=0.7, r_{2}=0.8, \alpha=0.01$, and $\beta=0.1$, the optimal feasible solution is obtained by iterative computation with a switching surface of $N_{0}=0.513$, which means that $\Sigma_{s 1}$ of the approach control mode will switch to $\Sigma_{s 2}$ of the smooth control mode when the distance between the optic lens and the desired position drops to $513 \mathrm{~nm}$. The corresponding controller parameters of the smooth mode controller are $K_{c}^{p 2}=0.0038121, K_{c}^{i 2}=0.0076242624$, and $K_{c}^{d 2}=-0.0060265$. Namely, the smooth control mode controller is represented as

$$
\begin{aligned}
C_{c}^{2}(z)= & 0.0038121+0.0076242624 \frac{z}{z-1} \\
& -0.0060265 \frac{z-1}{z} .
\end{aligned}
$$

The results of the closed-loop system with the switched PID controller are presented in Figures 6 and 7. The control signal of the switched PID controller, which provided the input voltage to the PZT actuator, is shown in Figure 6, and the corresponding output of the closed-loop system is shown in Figure 7. It can be seen that the whole setpoint process in the nanopositioning system has been divided into two stages by the switching point at $513 \mathrm{~nm}$ from the sample to the optic lens. Compared with the previous $\mathrm{H}_{2}$ optimized controller, the switched control method not only restricts the large overshoot of the closedloop system to avoid the collision but also possesses a high convergence speed. They have the similar fast-response ability, but the switched controller possesses the main advantage to limit the overshoot. The experimental results

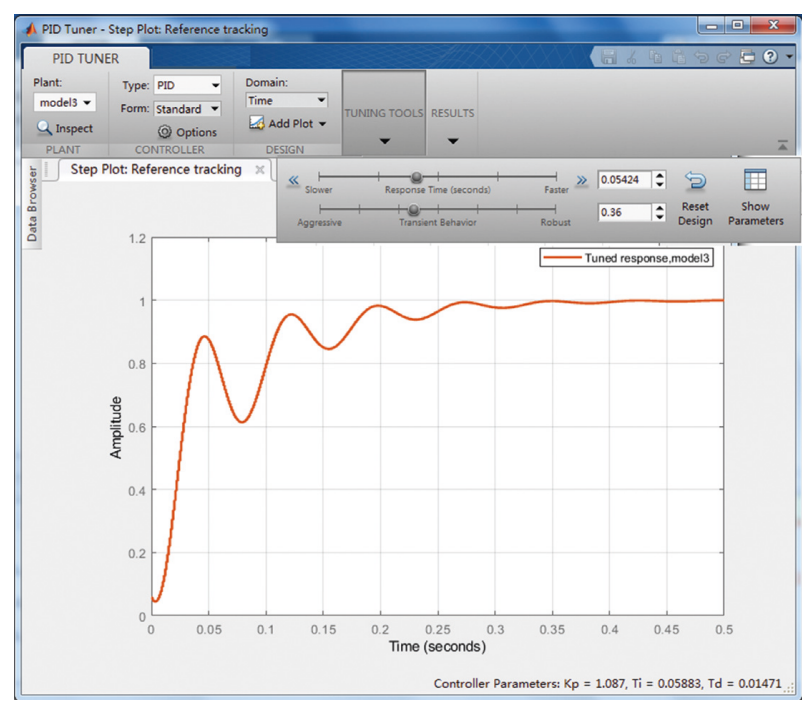

Figure 3: The output response of the closed-loop system with a single PID controller.

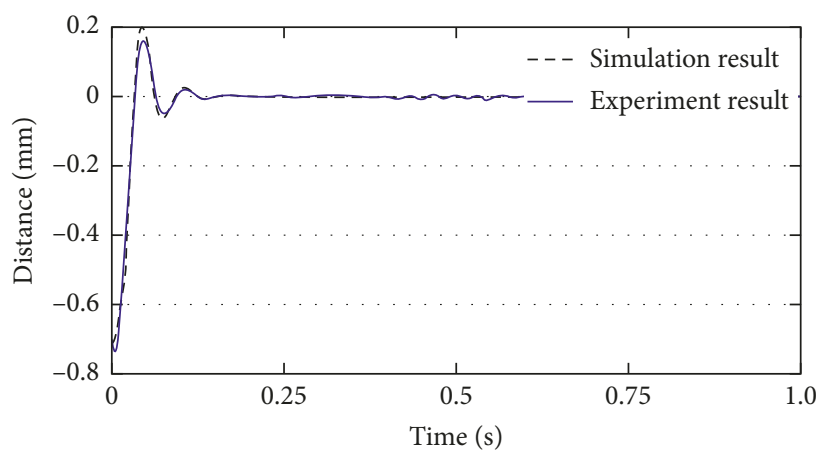

FIgURE 4: The output response of the closed-loop system with the $\mathrm{H}_{2}$ optimized controller.

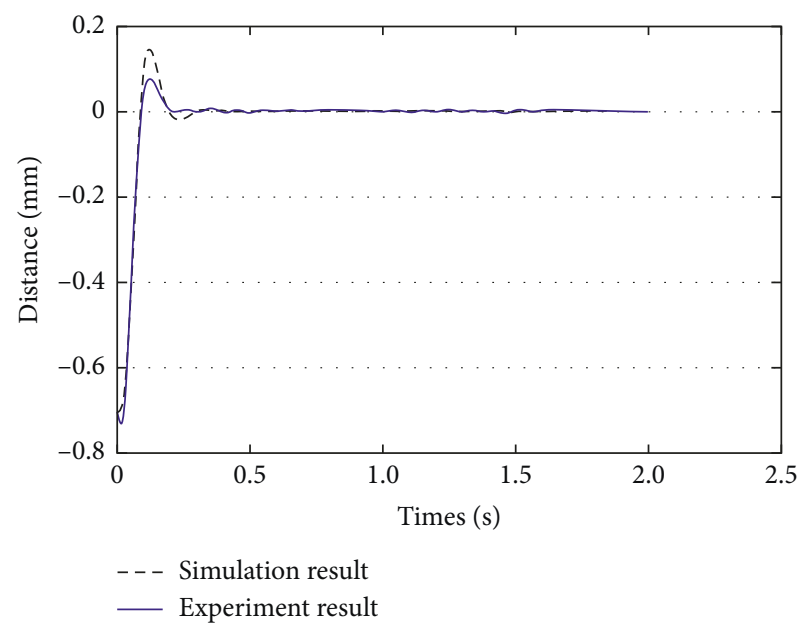

FIgURE 5: The output response of the closed-loop system with the approach mode PID controller.

indicate that the proposed switched PID controller can be used in the nanopositioning system for a fast approaching operation. 


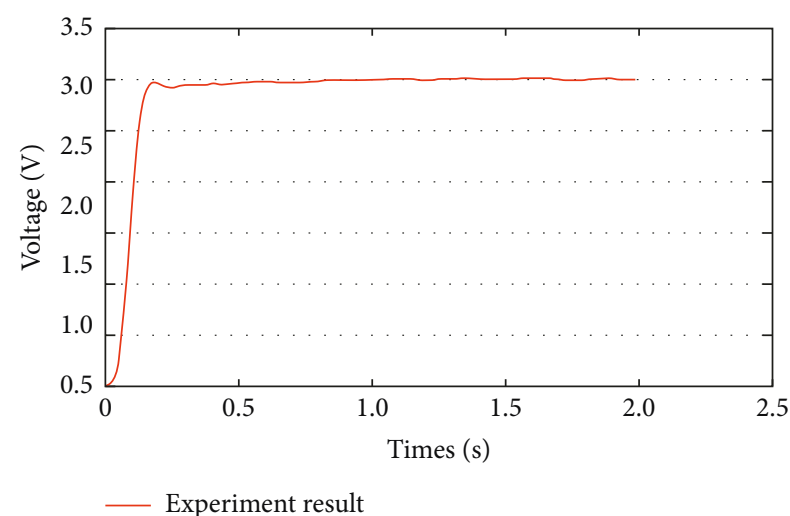

FIgUre 6: The input voltage of the PZT actuator with the switched PID controller.

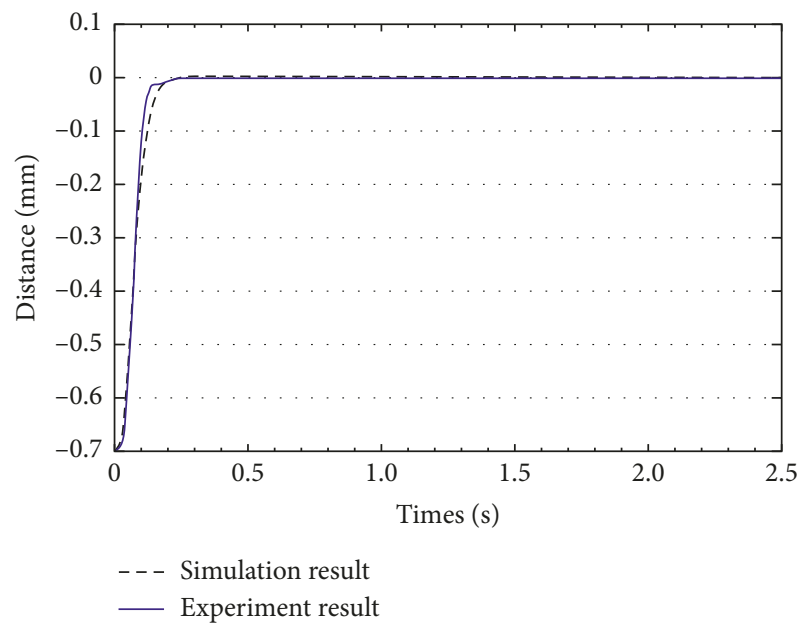

FIGURE 7: The output response of the closed-loop system with the switched PID controller.

\section{Conclusions}

In this paper, a switched PID controller-based fast setpoint control method has been developed for the nanopositioning system. The overshoot constraint of the fast setpoint process in the switched closed-loop system is investigated. The corresponding switched controllers can be determined based on solving the properly formulated synthesis algorithm with an optimized performance under the overshoot constraints. The performance of the switched PID controller is experimentally evaluated using a closed-loop nanopositioning system driven by a PZT actuator. The experimental results demonstrated the effectiveness of the switched PID control method to achieve a fast setpoint operation with a limited overshoot.

\section{Data Availability}

The data used to support the findings of this study are included within the article.

\section{Disclosure}

Part of the manuscript was presented in the "2017 IEEE 2nd Advanced Information Technology, Electronic and Automation Control Conference (IAEAC), Chongqing, China.”

\section{Conflicts of Interest}

The authors declare that there are no conflicts of interest.

\section{Acknowledgments}

This work was supported by the National Natural Science Foundation of China (51675321), the Doctoral Research Fund of Zhengzhou University of Light Industry (No. 2019BSJJ003), and the Shanghai Municipal Natural Science Foundation (15ZR1415800).

\section{References}

[1] Y. Hu and Y. Meng, "Numerical modeling and analysis of plasmonic flying head for rotary near-field lithography technology," Friction, vol. 6, no. 4, pp. 443-456, 2018.

[2] T. Chen, K. Meng, Z. Yang et al., "Scanning liquid-immersed microsphere optical superresolution imaging based on microrobotics manipulation," IEEE Transactions on Nanotechnology, vol. 17, no. 4, pp. 860-864, 2018.

[3] X. Shi, N. Coca-López, J. Janik, and A. Hartschuh, "Advances in tip-enhanced near-field Raman microscopy using nanoantennas," Chemical Reviews, vol. 117, no. 7, pp. 4945-4960, 2017.

[4] S. Oh, J. Jang, and J. W. Hahn, "Relative positioning method for near-field beam spot array with optical microscope image of lithographic patterns using linear regression," Applied Optics, vol. 56, no. 5, pp. 1346-1352, 2017.

[5] T. Tuma, W. Haeberle, H. Rothuizen, J. Lygeros, A. Pantazi, and A. Sebastian, "Dual-stage nanopositioning for high-speed scanning probe microscopy," IEEE/ASME Transactions on Mechatronics, vol. 19, no. 3, pp. 1035-1045, 2014.

[6] J. Berthelot, S. S. Aćimović, M. L. Juan, M. P. Kreuzer, J. Renger, and R. Quidant, "Three-dimensional manipulation with scanning near-field optical nanotweezers," Nature Nanotechnology, vol. 9, no. 4, pp. 295-299, 2014.

[7] J. Zemła, J. Danilkiewicz, B. Orzechowska, J. Pabijan, S. Seweryn, and B. Lekka, "Atomic force microscopy as a tool for assessing the cellular elasticity and adhesiveness to identify cancer cells and tissues," Seminars in cell \& developmental biology, vol. 73, pp. 115-124, 2018.

[8] Y. Lee, Y. E. Lee, K. H. Kim et al., "Effect of air bearing surface on shock resistance of optical head for solid immersion lens based near field recording system," IEEE Transactions on Magnetics, vol. 45, no. 5, pp. 2236-2239, 2009.

[9] Y. Lee, Y. E. Lee, S. J. Lee et al., "Improvement of shock resistance for solid immersion lens-based near field recording system using air-bearing surface," Japanese Journal of Applied Physics, vol. 49, no. 8, article 08KC05, 2010.

[10] D. Koide, T. Kajiyama, H. Tokumaru et al., "High-speed and precise gap servo system for near-field optical recording," Japanese Journal of Applied Physics, vol. 51, no. 8S2, article 08JA04, 2012. 
[11] J.-G. Kim, H.-W. Hwang, K.-S. Park et al., "Improved air gap control with acceleration feedforward controller using time delay for solid immersion lens-based near-field storage system," IEEE Transactions on Magnetics, vol. 47, no. 3, pp. 556-559, 2011.

[12] Y. K. Yong and A. J. Fleming, "High-speed vertical positioning stage with integrated dual-sensor arrangement," Sensors and Actuators A: Physical, vol. 248, pp. 184-192, 2016.

[13] X. Wen, L. M. Traverso, P. Srisungsitthisunti, X. Xu, and E. E. Moon, "High precision dynamic alignment and gap control for optical near-field nanolithography," Journal of Vacuum Science \& Technology B, Nanotechnology and Microelectronics: Materials, Processing, Measurement, and Phenomena, vol. 31, no. 4, article 041601, 2013.

[14] J. Li and L. Yang, "Adaptive pi-based sliding mode control for nanopositioning of piezoelectric actuators," Mathematical Problems in Engineering, vol. 2014, Article ID 357864, 10 pages, 2014.

[15] H. Hwang, J. G. Kim, K. W. Song et al., "High-speed gap servo control for solid-immersion-lens-based near-field recording system with a flexible optical disk," Japanese Journal of Applied Physics, vol. 50, no. 9S1, article 09MC04, 2011.

[16] Y. K. Yong, S. O. R. Moheimani, B. J. Kenton, and K. K. Leang, "Invited review article: high-speed flexure-guided nanopositioning: mechanical design and control issues," Review of Scientific Instruments, vol. 83, no. 12, article 121101, 2012.

[17] T. Chen and B. A. Francis, Optimal Sampled-Data Control Systems, Springer Science \& Business Media, Berlin, Germany, 2012.

[18] H. Liu, Y. Li, Y. Zhang et al., "Intelligent tuning method of PID parameters based on iterative learning control for atomic force microscopy," Micron, vol. 104, pp. 26-36, 2018.

[19] S. Kuiper and G. Schitter, "Model-based feedback controller design for dual actuated atomic force microscopy," Mechatronics, vol. 22, no. 3, pp. 327-337, 2012.

[20] J. G. Kim, M. S. Kang, W. H. Shin et al., "Improved gap servo system using a brake pulse for a SIL-based near-field recording system," Microsystem Technologies, vol. 16, no. 1-2, pp. 309-315, 2010.

[21] S. Zimmermann, T. Tiemerding, T. Li, W. Wang, Y. Wang, and S. Fatikow, "Automated mechanical characterization of 2D materials using SEM based visual servoing," International Journal of Optomechatronics, vol. 7, no. 4, pp. 283-295, 2013.

[22] H. Zhu, C. K. Pang, and T. J. Teo, "A flexure-based parallel actuation dual-stage system for large-stroke nanopositioning," IEEE Transactions on Industrial Electronics, vol. 64, no. 7, pp. 5553-5563, 2017. 


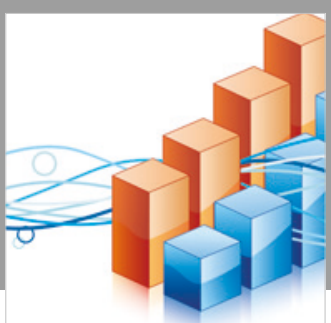

Advances in

Operations Research

\section{-n-m}
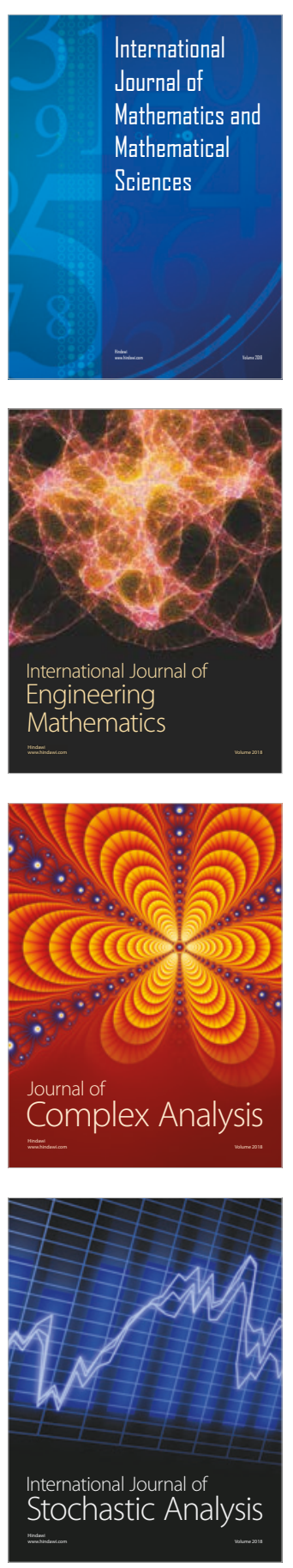
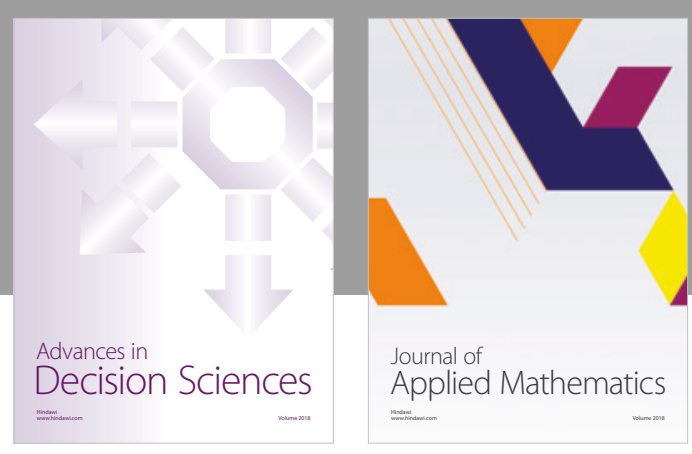

Journal of

Applied Mathematics
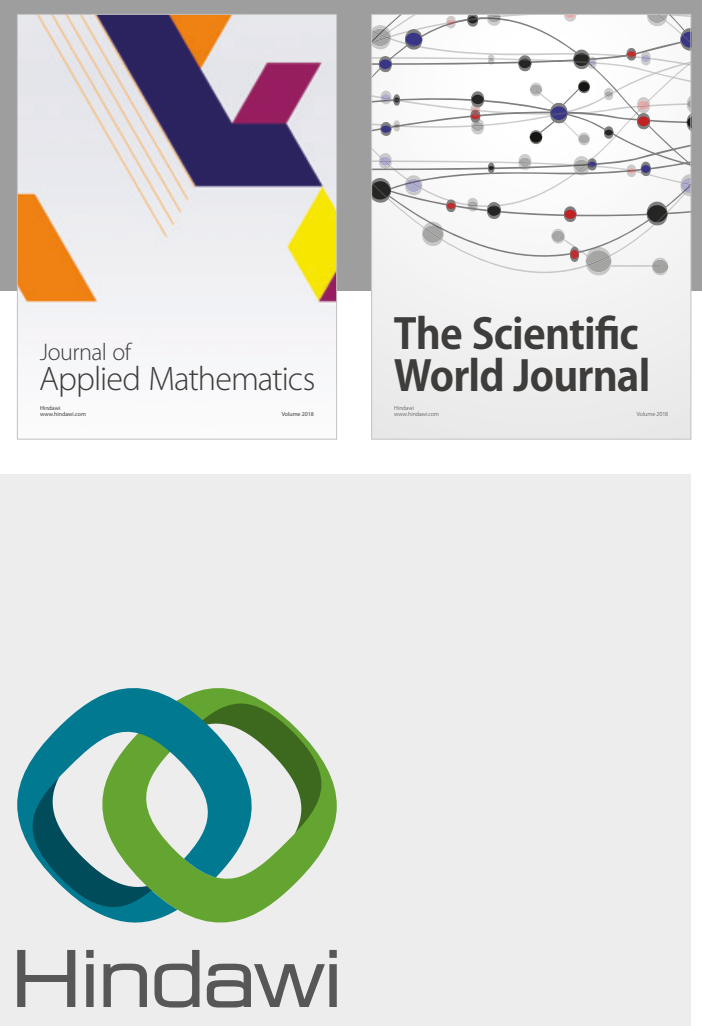

Submit your manuscripts at

www.hindawi.com

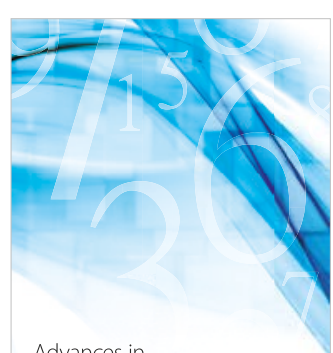

Advances in
Numerical Analysis
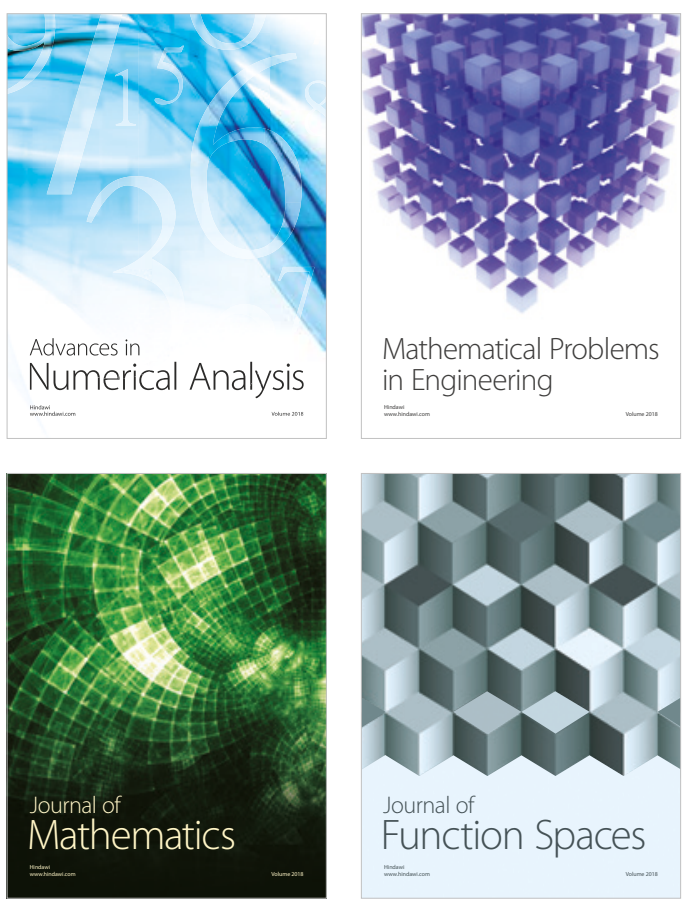

Mathematical Problems in Engineering

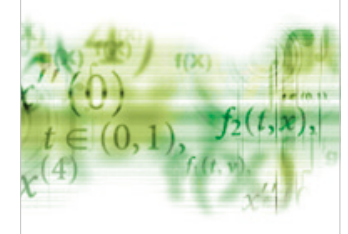

International Journal of

Differential Equations

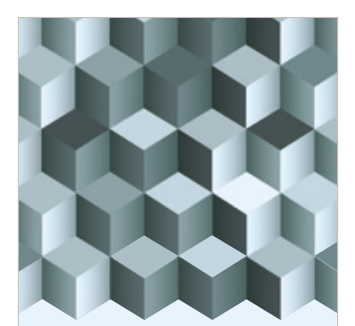

Journal of

Function Spaces
The Scientific

World Journal

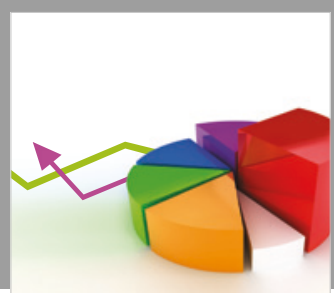

Journal of

Probability and Statistics
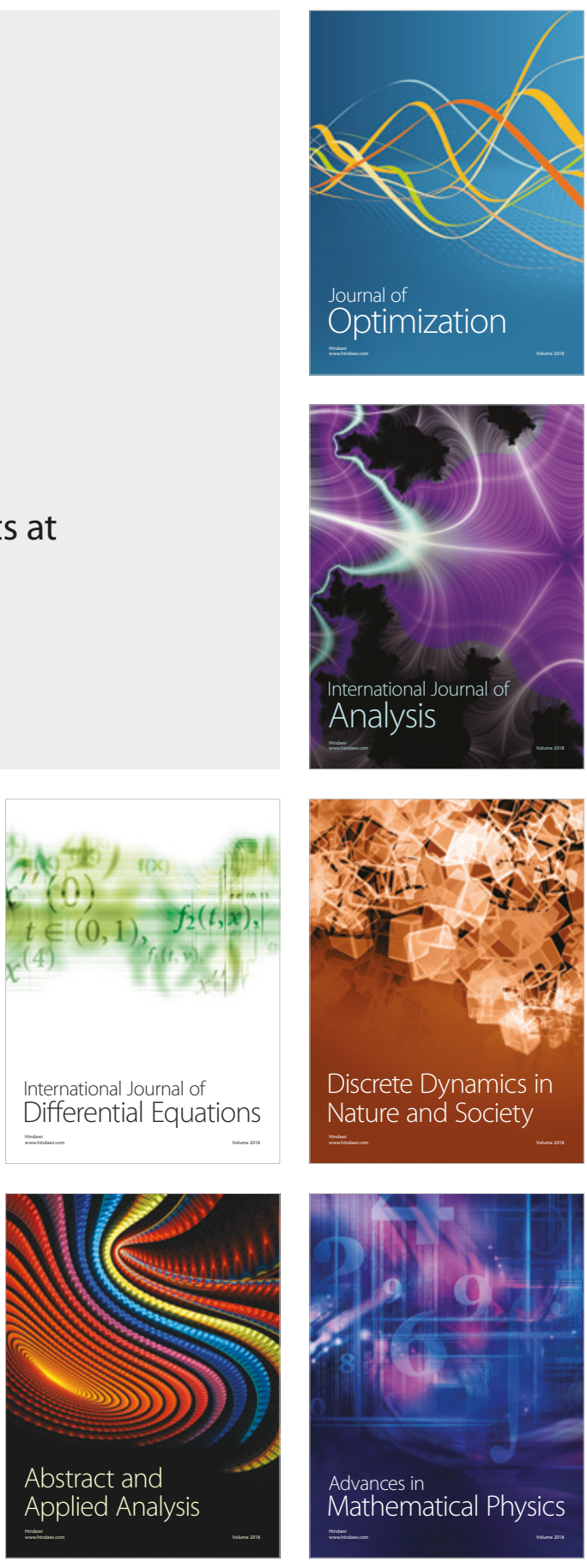